\title{
A Minimum Distance guided Genetic Algorithm for Multi-User Detection in a Multi-Carrier CDMA Wireless Broadband System
}

\author{
Qiang Ni, Jehanzeb Jehanzeb, Yang Zhang, Sheng-Uei Guan \\ Electronic \& Computer Engineering, School of Engineering and Design, Brunel University, West London, UK \\ Correspondence to: Qiang.Ni@brunel.ac.uk
}

\begin{abstract}
We propose a novel Minimum Distance guided Genetic Algorithm (MDGA) for Multi-User Detection (MUD) in a synchronous Multi-Carrier Code Division Multiple Access (MC-CDMA) broadband wireless system. In contrast to conventional GAs, our MDGA exploits adequately the output from a bank of Matched Filters as guidance. It starts with a balanced ratio of exploration and exploitation which is maintained throughout the process. A novel replacement strategy is proposed which increases dramatically the convergence rate as compared to the conventional GAs. This allows us to use the simplest form of genetic operators to gain significant reduction in computational complexity as well as near-optimum results. The simulation results demonstrate that our scheme achieves $\mathbf{9 9 . 5 4 \%}$ and $50+\%$ reduction in computational complexity as compared to the MUD schemes using exhaustive search and conventional GA respectively.
\end{abstract}

Index Terms - MC-CDMA, Multi-User Detection, Genetic Algorithm, Multiple Access Interference

\section{INTRODUCTION}

Multi-Carrier Code Division Multiple Access (MC-CDMA) is an emerging broadband wireless transmission technique that combines the advantages of both Orthogonal Frequency Division Multiplexing (OFDM) and Code Division Multiple Access (CDMA) together [1-3]. In MC-CDMA, orthogonal spreading chips from different users are modulated over all the available sub-carriers to achieve high frequency diversity gain. Multi-path problem can be mitigated effectively by using multi-carrier OFDM modulation technique. This system allows all the users to simultaneously share the same frequency band by transmitting data on all sub-carriers at a same time. However, MC-CDMA suffers from a so-called Multiple Access Interference (MAI) problem because in reality spreading codes are not always perfectly orthogonal. To reduce MAI, Verdu [5] proposed a joint detection of all users in CDMA system, called multi-user detection (MUD). The idea of MUD is to treat MAI as a part of information, not noise. In this way, it could improve the system performance significantly. Hence, MUD has received much attention for both CDMA and emerging MC-CDMA systems [4-8].

However, the optimal MUD detector proposed by Verdu becomes impractical due to high computational complexity when there is a large number of users. With the optimal MUD detector, the receiver should consider all possible combinations of the transmitted signal and check which one could maximize the joint correct decision probability of the bits received from all the users. This is not feasible since the system has to check every possible combination of the bit vector sent. For example, if there are $K$ users in the system, the total number of possibilities becomes $2^{K}$. Hence, the computational complexity of the optimal detector increases exponentially with the increasing number of users. Therefore, recent research work focuses on looking for suboptimal MUD solutions which are feasible in practice.

Genetic Algorithm (GA) [9] is an intelligent technique derived from the principles of genetics and natural selection which can be used to search suboptimal solution with low complexity for multi-objective combinatory optimization problem. GA based suboptimal MUD was first proposed in [10] for CDMA systems showing its capability in approaching single-user performance bound at lower computational complexity than the exhaustive search used in the optimal detector. Later some other GA based MUDs have been proposed (e.g. $[1,11])$ with a much better start than that in [10] but gradual convergence towards the optimum solution due to their classic sophisticated replacement strategies. Therefore, a simple intelligent scheme is still required to improve the convergence rate without increasing the complexity of the system. In this paper we introduce a novel and simple search mechanism to increase significantly the convergence speed of conventional GA schemes (e.g. [10,11]) for MUD application.

The rest of the paper is organized as follows. Section II describes the system model for synchronous MC-CDMA system. The Minimum Distance guided GA is proposed in section III, followed by its performance analysis in section IV. Section V concludes the paper.

\section{SYNCHRONOUS MC-CDMA SYSTEM MODEL}

We consider a bit-synchronous MC-CDMA system as shown in Fig. 1, where $K$ users simultaneously transmit data bits over an additive white Gaussian noise (AWGN) channel. Assuming that the number of chips is equal to the number of sub-carriers, each user bit is firstly spread by a unique pseudo-random 
sequence. Then each chip of the corresponding spread signal is modulated over each of the " $\mathrm{M}$ " orthogonal sub-carriers. The $k^{\text {th }}$ user's transmitted signal is given by,

$$
s_{k}(t)=\sqrt{\frac{2 E_{k}}{M}} \sum_{m=1}^{M} c_{k, m}(t) \cdot b_{k}(t) e^{j \omega_{m} t}
$$

where, $\mathrm{E}_{\mathrm{k}}$ : The transmit power of the $k^{\text {th }}$ user $(k=1, \ldots, K)$,

$M$ : Number of sub-carriers,

$\mathrm{T}_{\mathrm{b}}$ : Data bit duration,

$\mathrm{b}_{\mathrm{k}}(t)$ : The transmitted bit sequence of the $k^{\text {th }}$ user,

$\mathrm{c}_{\mathrm{k}, \mathrm{m}}(t)$ : Time-domain representation of the $k^{\text {th }}$ user's spreading sequence over the $m^{\text {th }}$ sub-carrier,

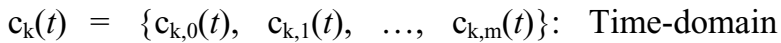
representation of the $k^{\text {th }}$ user's spreading code over all sub-carriers,

$\omega_{\mathrm{m}}=2 \pi \mathrm{f}_{\mathrm{m}}$, where $\mathrm{f}_{\mathrm{m}}(\mathrm{m}=1, \ldots, \mathrm{M})$ are the subcarrier frequencies and $\mathrm{f}_{\mathrm{m}}=\mathrm{f}_{1}+(\mathrm{m}-1) \Delta \mathrm{f}$, where frequency spacing $\Delta \mathrm{f}$ is $1 / \mathrm{T}_{\mathrm{b}}$.

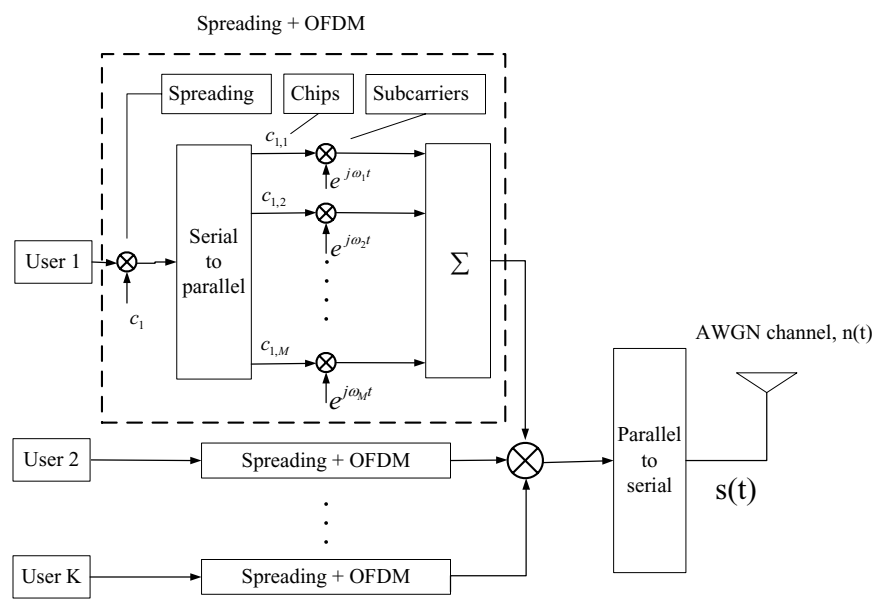

Fig. 1. Transmitter model of MC-CDMA under AWGN uplink channel.

After the spreading and OFDM modulation, the signal is then transmitted over the AWGN channel. Hence, a number of users are able to share all the bandwidth (sub-carriers) simultaneously. The energy of the spreading sequence over a bit period of " $T_{b}$ " for each user is assumed to be " 1 ". Note that the spreading sequences used may not be perfectly orthogonal to each other in reality due to the cross-correlation product. Assuming perfect synchronization at the receiver, they will have good auto-correlation properties but bad cross-correlation properties.

Fig. 2 shows a simple MC-CDMA receiver followed by a novel GA based MUD. The data carried by each sub-carrier is firstly de-modulated. The demodulated data on the sub-carrier frequency $f_{1}$ yields a composite part of the spread signals of all the users, contributed by the $1^{\text {st }}$ chips of their respective spreading sequences. Similarly, for the sub-carriers $f_{2}$ till $f_{M}$, the contribution is from the set of the $2^{\text {nd }}$ chips till the last set of chips of the spreading sequences from all users respectively. The received signal on the $\mathrm{m}^{\text {th }}$ sub-carrier is,

$$
r_{m}(t)=\sum_{k=1}^{K}\left[\sqrt{\frac{2 E_{k}}{M}} c_{k, m}(t) \cdot b_{k}(t)\right]+n(t)
$$

where, $\mathrm{n}(\mathrm{t})$ is the AWGN noise added to the signal with two-sided power spectrum density $\mathrm{N}_{\mathrm{o}} / 2$.

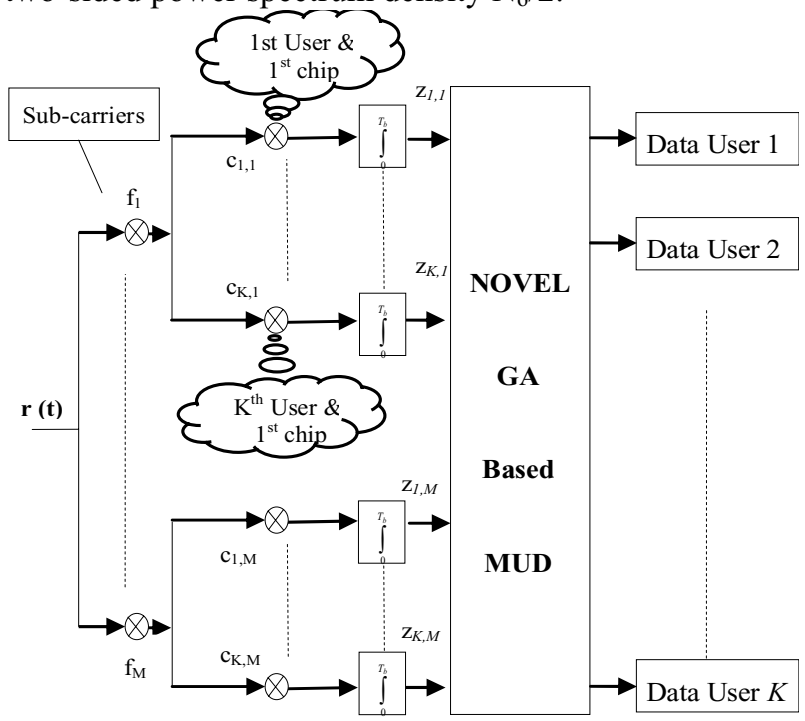

Fig. 2. MC-CDMA receiver model for uplink.

The information of any particular bit of a user is carried by all of the sub-carriers at any given time. The matrix representation of the received signal on the $\mathrm{m}^{\text {th }}$ sub-carrier is as follows:

$$
\boldsymbol{r}_{\mathrm{m}}=\boldsymbol{C}_{\mathrm{m}} \boldsymbol{E} \boldsymbol{b}+\boldsymbol{n}
$$

where,

$$
\begin{aligned}
& \boldsymbol{C}_{\mathrm{m}}=\left[\mathrm{c}_{1, \mathrm{~m}}, \cdots, \mathrm{c}_{\mathrm{K}, \mathrm{m}}\right\rfloor \\
& \boldsymbol{E}=\operatorname{diag}\left[\sqrt{\frac{2 \mathrm{E}_{1}}{\mathrm{M}}}, \cdots, \sqrt{\frac{2 \mathrm{E}_{\mathrm{K}}}{\mathrm{M}}}\right] \\
& \boldsymbol{b}=\left[\mathrm{b}_{1}, \cdots, \mathrm{b}_{\mathrm{K}}\right]^{\mathrm{T}} \\
& \boldsymbol{n}=\left[\mathrm{n}_{1}, \cdots, \mathrm{n}_{\mathrm{K}}\right]^{\mathrm{T}}
\end{aligned}
$$

As shown in Fig. 2, the output from the matched filters can be represented in a form of vector " $\boldsymbol{Z}_{\mathrm{m}}$ " [1]:

$$
\boldsymbol{Z}_{\mathrm{m}}=\left[\mathrm{z}_{1, \mathrm{~m}}, \cdots, \mathrm{z}_{\mathrm{K}, \mathrm{m}}\right]=\boldsymbol{R}_{\mathrm{m}} \boldsymbol{E} \boldsymbol{b}+\boldsymbol{n}
$$

where, $\quad \boldsymbol{R}_{m}=\left[\begin{array}{cccc}\rho_{11}^{(m)} & \rho_{12}^{(m)} & \cdots & \rho_{1 K}^{(m)} \\ \rho_{21}^{(m)} & \rho_{22}^{(m)} & \cdots & \rho_{2 K}^{(m)} \\ \vdots & \vdots & \vdots & \vdots \\ \rho_{K 1}^{(m)} & \rho_{K 2}^{(m)} & \cdots & \rho_{K K}^{(m)}\end{array}\right]$

$\rho_{j k}^{(m)}$ is actually the value of cross-correlation $(j \neq k)$ and the auto-correlation $(j=k)$ of the spreading sequences contributing on the $\mathrm{m}^{\text {th }}$ sub-carrier. MAI imposed by the cross-correlation between the spreading codes is the main problem in an MC-CDMA system. The Matched Filter Conventional Detector (CD) for the MC-CDMA system treats MAI as noise. However if we exploit knowledge of the users' spreading sequences as auxiliaries, the MAI can be substantially reduced [1], resulting 
in the corresponding user capacity gains and better BER performance.

The joint optimum decision rule for a $\mathrm{K}$-user synchronous CDMA system was discussed in [1]. That model can be easily extended to a K-user MC-CDMA system model. The discrete-time correlation metric on the $\mathrm{m}^{\text {th }}$ sub-carrier can be expressed in vector form as:

$$
\Omega_{m}(\boldsymbol{b})=2 \operatorname{Re}\left[\boldsymbol{b}^{T} \boldsymbol{E} \boldsymbol{Z}_{m}\right]-\boldsymbol{b}^{T} \boldsymbol{E} \boldsymbol{R}_{m} \boldsymbol{E} \boldsymbol{b}
$$

Since the data is transmitted over M orthogonal sub-carriers, the contribution of all the likelihood functions will yield an optimum vector $\hat{\boldsymbol{b}}$

$$
\Omega(\boldsymbol{b})=\sum_{m=1}^{M} \Omega_{m}(\boldsymbol{b})=\sum_{m=1}^{M}\left\{2 \operatorname{Re}\left[\boldsymbol{b}^{T} \boldsymbol{E} \boldsymbol{Z}_{m}\right]-\boldsymbol{b}^{T} \boldsymbol{E} \boldsymbol{R}_{m} \boldsymbol{E} \boldsymbol{b}\right\}
$$

Hence, the decision rule for the optimum K-user MC-CDMA multi-user detection scheme based on the maximum likelihood criterion is to choose the specific bit combination $\boldsymbol{b}$ out of $2^{K}$ possible combinations, that maximizes the correlation metric of Equation (7). Therefore, it is

$$
\hat{\boldsymbol{b}}=\arg \left\{\max _{\boldsymbol{b}}[\Omega(\boldsymbol{b})]\right\}
$$

In the next section, we propose a novel Minimum Distance guided GA for MUD (MDGA-MUD) which provides fast convergence towards near-optimum solutions with low computational complexity.

\section{Minimum Distance Guided Genetic Algorithm}

Our proposed novel GA is designed to accelerate the searching speed to find the optimal solution by adequately exploiting the output of a bunch of CDs as guidance. As compared to conventional GA mechanisms for MUD [1, 10, 11], our MDGA proposes two main modifications, M1 and M2. The idea is to keep a balanced search throughout the GA generations: M1 allows us to start the initial search guided by CD's output together with a reasonable amount of randomness derived from general GA theory. M2 is an intelligent replacement strategy that plays the dominant role in providing fast convergence rate. Combining the two modifications together offers our GA a good jumping-off point of search as well as increased convergence with low complexity while keeping the optimum performance. Next, we explain in detail how the MDGA operates.

\section{A. Initial Population (M1)}

This section explains the first modification - named M1 in this paper. Considering that a good initial estimation of the possible solutions is worthful for GA to obtain good performance at low cost of searching progress, the selection of initial population to approach optimal solution is created by mutating the output of a CD.

Let the total population of chromosomes be " $\mathrm{N}_{\mathrm{p}}$ ", where each chromosome is represented as each row vector in the population matrix. In order to ensure that each bit of a CD's output experiences change at least once, we generate the initial "K +1 " set of population by perturbing the CD's output in such a way that the Hamming Distance (the number of different bits) between the CD's output and the new individual remains 1, which is the essence of our so-called Minimum Distance approach. The CD's output is considered as a robust jumping-off point of search. However, there is a high probability that a search which is guided only by CD's output can get stuck at local optima at high SNR values. In order to ensure a certain proportional searching diversity we make the rest of the population randomly to explore optimum solution in the search space as shown in Fig. 3.

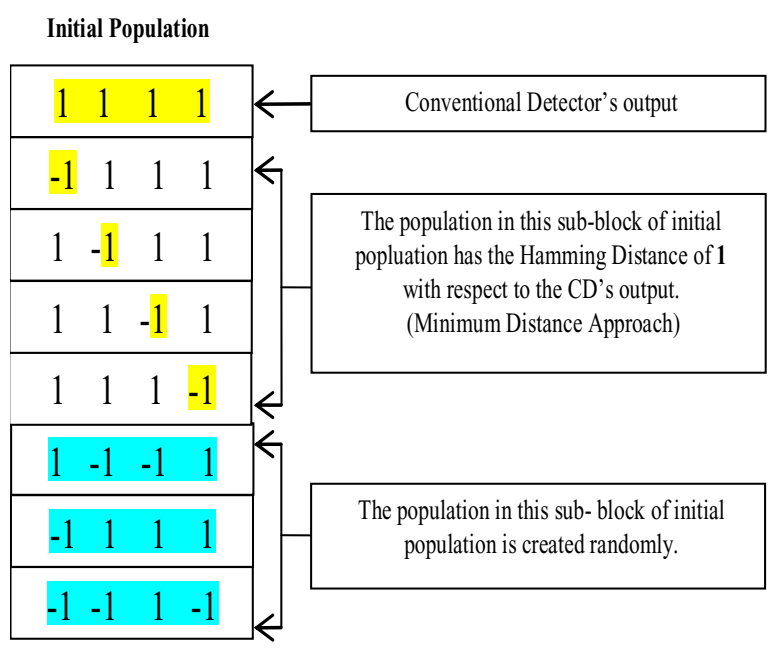

Fig. 3. Formation of Initial pool for the proposed GA.

It is noted here that this modification in the formation of initial population will create some dependency of the population size on the number of users. For example, if the number of users is " $\mathrm{K}$ ", then the population size should be chosen in a range of $K<N_{P} \leqslant 2 K$. So, the algorithm will adjust the population size with the increasing number of users which is actually in accordance with the study carried out by [1]; indicating the need of properly increasing the population size when the number of users in the system is increased.

\section{B. Fitness Function}

In general GA, the goal of a fitness function is to evaluate the worth or status of each chromosome. In the MUD problem, the objective of the fitness function is the maximization of the cost (or objective) function, as defined in Equation (7). Each bit in vector "b" actually represents a bit sent by a particular user. The task of MUD is to detect all the users' bits coherently. In order to correctly detect the bit of each user, we need to find such a combination of vector "b" that will maximize the cost function. Hence, GA is invoked in a search for such an optimum solution vector $\hat{b}$.

\section{Replacement Strategy (M2)}

After the successful evaluation of the initial pool, truncation selection takes place in which the candidate solutions are ordered by fitness, and according to some selection rate, 
"Xrate". In our case, we set a fixed value Xrate $=1 / 2$. The most $\left\lfloor N_{p} / 2\right\rfloor$ fittest individuals in the rank list are selected as candidate parents, where $\lfloor\bullet\rfloor$ stands for floor operator. For example, if the population size is 30 , we first select 15 best candidates by the truncation-selection method and then starting from the fittest two parents we form two offspring by performing simple one-point crossover operation. Then, from the next two fittest mating parents we form two more offspring and so on till we form $\mathrm{N}_{\mathrm{P}}-(\mathrm{K}+1)=30-(15+1)=14$ new offspring. We designed the mating process in a way that in each generation, the number of offspring generated is $\mathrm{K}+1-\left\lceil\mathrm{N}_{\mathrm{p}} / 2\right\rceil$ less than the selected parents, where symbol $\left\lceil\mathrm{N}_{\mathrm{p}} / 2\right\rceil$ acquires the ceiling value of $\mathrm{N}_{\mathrm{p}} / 2$.

After successful mating, we replace $\mathrm{K}$ prior chromosomes in the rank except fittest mate from the population matrix by performing the same Minimum Distance approach using the fittest mate, i.e. the population matrix will contain the fittest mate on the top along with the " $\mathrm{K}$ " individuals having Hamming Distance of $\mathbf{1}$ biased from the fittest and the previously formed " $\mathrm{N}_{\mathrm{p}}-(\mathrm{K}+1)$ " number of offspring. This is how we exploit the information of the best mate (local search) at each generation and how we create a reasonable number of offspring out of the mating pool for exploration.

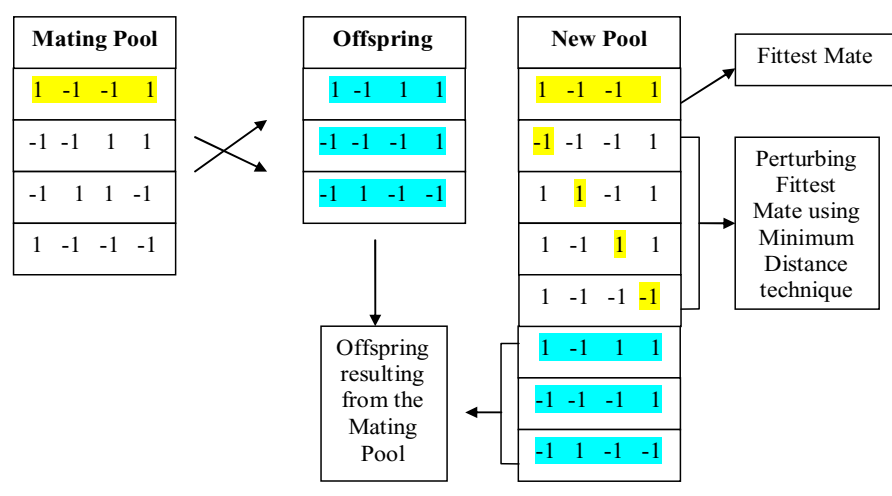

Fig. 4. A Novel Replacement Strategy for MDGA.

In MDGA, the GA is firstly guided by CD's output and then in the following generations, it is guided by the best mate created from the population matrix in each generation. The fittest-initiated " $\mathrm{K}+1$ " individuals along with the " $\mathrm{N}_{\mathrm{p}}-(\mathrm{K}+1)$ " offspring generated from the mating pool ensures that the GA would not likely be stuck at the local maximum. The process involved in the replacement strategy is shown in the Fig. 4, with an example population size of 8 chromosomes for a 4-user MC-CDMA system. Note that the crossover point is taken after the 2nd bit.

\section{Type of Mutation \& Crossover}

The new population matrix undergoes mutation process which further ensures that our GA search would not get stuck around local maxima. Usually, the mutation probability is chosen closer to 0 (in our case, it is chosen to be 0.1 which gives the best result from a serial of pretests).

The crossover operator is chosen to be the simplest of its nature i.e. one-point crossover to reduce computational complexity as compared to conventional GA [11] that uses double or uniform crossover. The crossover probability is chosen to be 1 . The population size after each generation remains constant reflecting the simplicity of the algorithm in its implementation.

\section{E. Elitism}

The Elitism property is invoked just like in any other conventional GA to preserve the superior chromosome of the fittest individual during mutation process. There is only one best chromosome to be found, and hence, the elitism property is invoked only for one fittest individual out of the total population in every generation. Fig. 5 shows the flow of operations in the proposed GA. In the next section we define the simulation parameters and show the performance and the convergence rate of the highlighted MDGA based MUD.

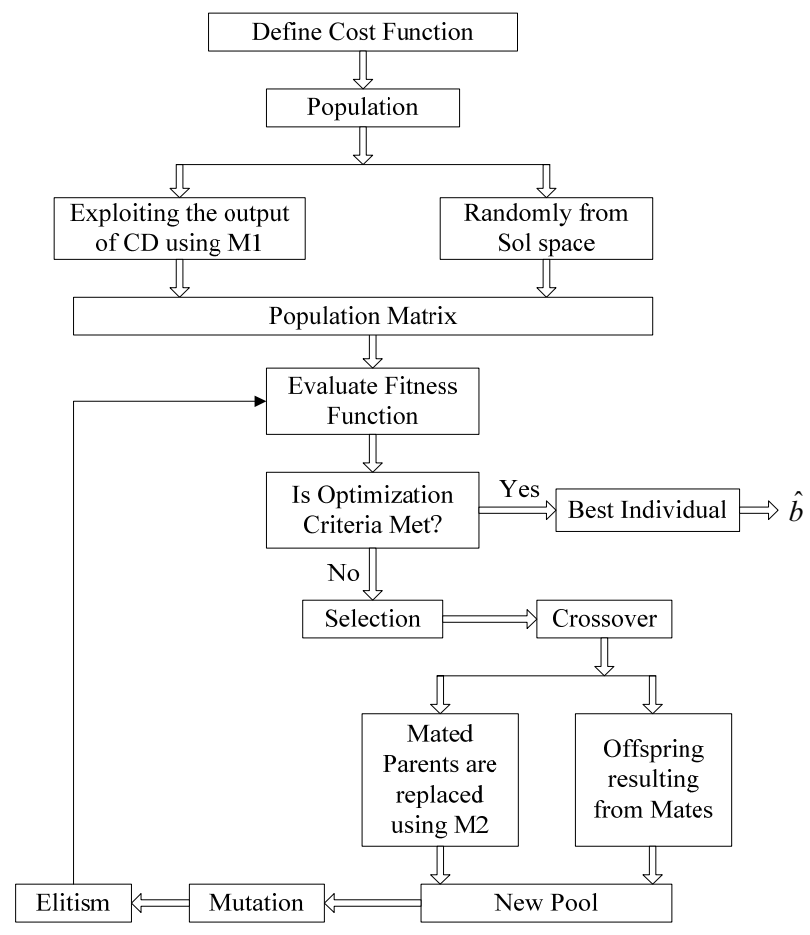

Fig. 5. Flow chart of MDGA-MUD

\section{Simulation Results}

The BER, optimum solution detection time and the computational complexity involved are the three main performance criteria to be satisfied. The detection time is governed by the number of generations required to obtain a reliable decision. The computational complexity is determined by the total number of objective function evaluations. Table I shows the configuration parameters used in the simulations. BPSK modulation type is chosen in the system.

As shown in Fig. 6, MDGA can converge very close to the optimum within only 5 generations with a population size of 30 when the number of users is 15 . This is because that MDGA can not only find a good start point but also achieve a high 
convergence rate with each generation due to the proposed two methods (M1 and M2). The number of cost function evaluations taken by MDGA is only 150 till it obtains near-optimum solutions.

TABLE I CONFIGURATION OF THE MDGA-MUD

\begin{tabular}{|c|c|}
\hline PARAMETERS & METHODS/VALUES \\
\hline Population size $\mathrm{N}_{\mathrm{p}}$ & 30 \\
\hline INITIALIZATION & Initial population generated using M1 \\
\hline Selection Method & Truncation Selection \\
\hline Selection rate & $50 \%$ \\
\hline Crossover operation & Single-point crossover \\
\hline Crossover Probability $\mathrm{p}_{\mathrm{c}}$ & 1 \\
\hline NEW POOL & New pool created using M2 \\
\hline Mutation operation & Standard binary mutation \\
\hline Mutation Probability $\mathrm{p}_{\mathrm{m}}$ & 0.1 \\
\hline Elitism & Yes \\
\hline Termination Generation & 5 \\
\hline Spreading Factor $(\mathrm{SF})$ & 15 \\
\hline
\end{tabular}

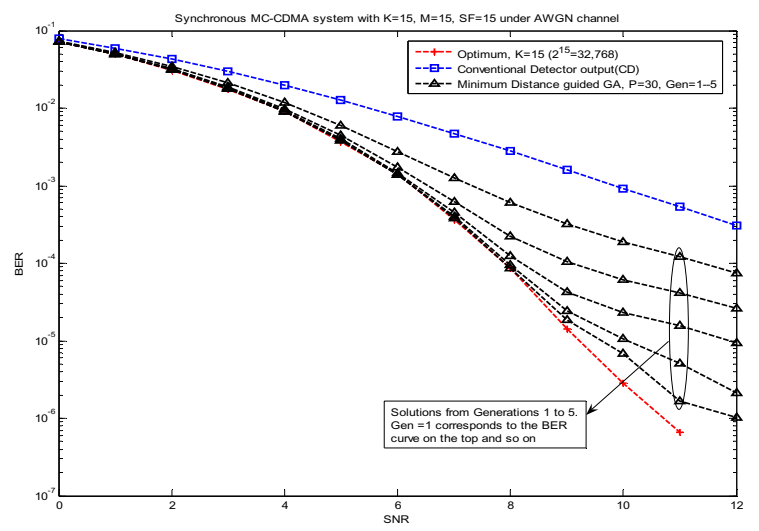

Fig. 6. The BER performance of the first 5 generations of MDGA assisted MUD in MC-CDMA over an AWGN channel. The MDGA-MUD can converge very close to the optimum in as low as 5 generations with a population size of 30 for a 15-user synchronous MC-CDMA system ( $\mathrm{SF}=15$ \& $\mathrm{M}=15)$.

In Fig 7, we investigate the individual impact of each modification. Although the Modification 2 (M2) has a poor start because of the conventional approach used in intializing the GA population which it actually happens in the first geneation, but it also provides great deal of convergence in the successive generations due to the novel replacement strategy used in MDGA. Whereas the Modification 1 (M1) has a good start due to the Minimum Distance aproach used to initiate the population and poor convergence due to the conventional replacement strategy. Their mutual contribution provides good convergence which is the essential of our proposed scheme.

When SNR increases, the MAI gets higher. This causes the output from CD further away from the optimum. In order to deal with such an issue, the MDGA introduces reasonable amount of randomness in the initial population in order to gain the diversity in the search space at high SNR values. Furthermore, it utilizes the information carried by the best individual in the population matrix in each generation so as to get better and better solutions. It also ensures that the presence of significant amount of diversity in the new population set by newly producing a number of offsprings from the selected mates. The new comers (offsprings) along with those individuals exploited best mate's by the Minimum Distance approach reacts in our GA to converge very fast towards the optimum. The conventional GA lacks this ability and is more likely to get stuck at local maxima and hence requires an increase in the number of generations before search termination.

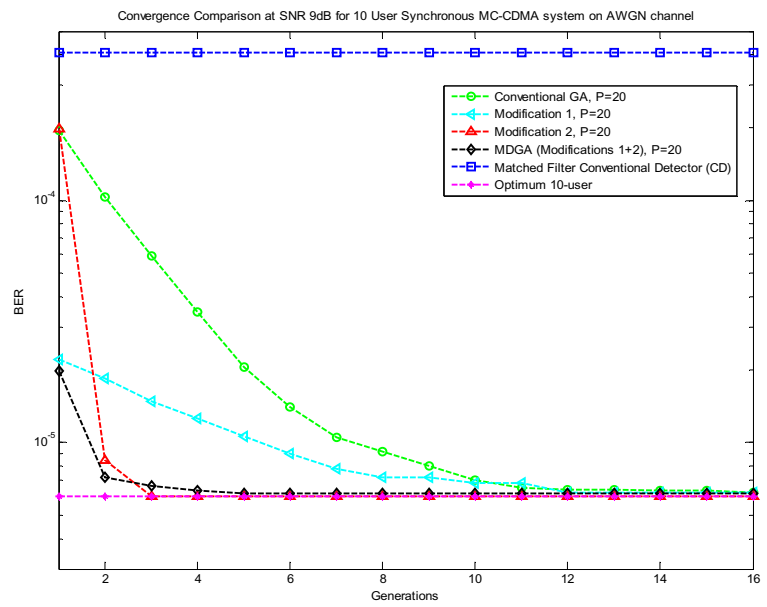

Fig. 7. Convergence comparison of MDGA vs. Conventional GA [11] for 10-User MC-CDMA system under 9dB SNR. It also shows the role played by each modiciation independently. Please note that the convergence graphs of the two modifications actually cross each other after the first generation.

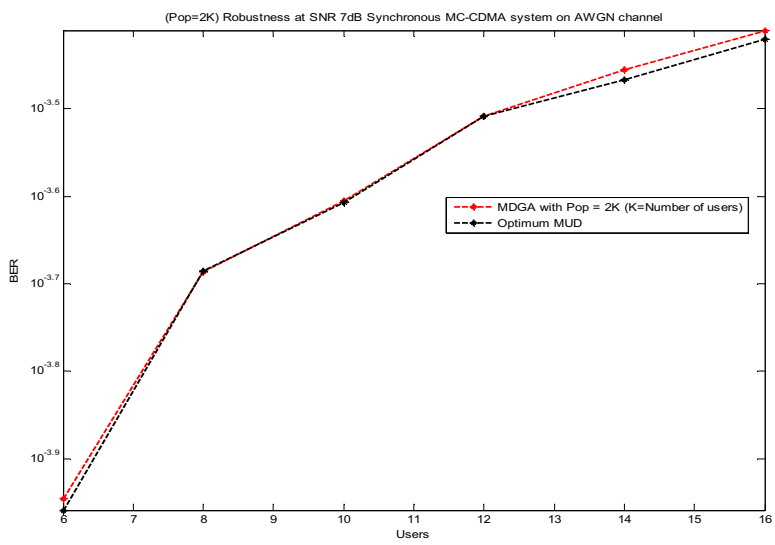

Fig. 8. MDGA is robust with the increasing number of users in the MC-CDMA system.

The population size in MDGA is dependent on the number of users " $\mathrm{K}$ " in the system. The simulation results in Fig. 8 show that with the increasing number of users in the system, MDGA still closely inosculates the optimum solution. This shows that the fixed relationship between Population size and the number of users $(\mathrm{Pop}=2 \mathrm{~K})$ with the Termination Generation $=5$ is true for all user scenarios and hence, our scheme is robust with the increasing number of users in the system.

In this paper, the computational complexity is defined as the number of cost function evaluations needed to reach the optimum solution [1]. The percentage of complexity involved 
is $\left(\frac{P o p \times G e n}{2^{K}}\right) \times 100 \%$. As shown in Fig. 9, MDGA can give the near-optimum solution within only 150 cost function evaluations $(\mathrm{Gen}=5$, Pop $=30)$ as compared to $2^{15}(32,768)$ evaluations taken by exhaustive search. Hence, it achieves 99.54\% reduction in complexity as compared to ML-MUD. Also from Fig. 7, as compared to conventional GA [11] with $240(\mathrm{Gen}=12, \mathrm{Pop}=20)$ cost function evaluations, the MDGA reaches the optimum in only $100(\mathrm{Gen}=5$, Pop $=20)$ cost function evaluations, ensuring $58.3 \%$ complexity reduction in terms of cost function evaluations.

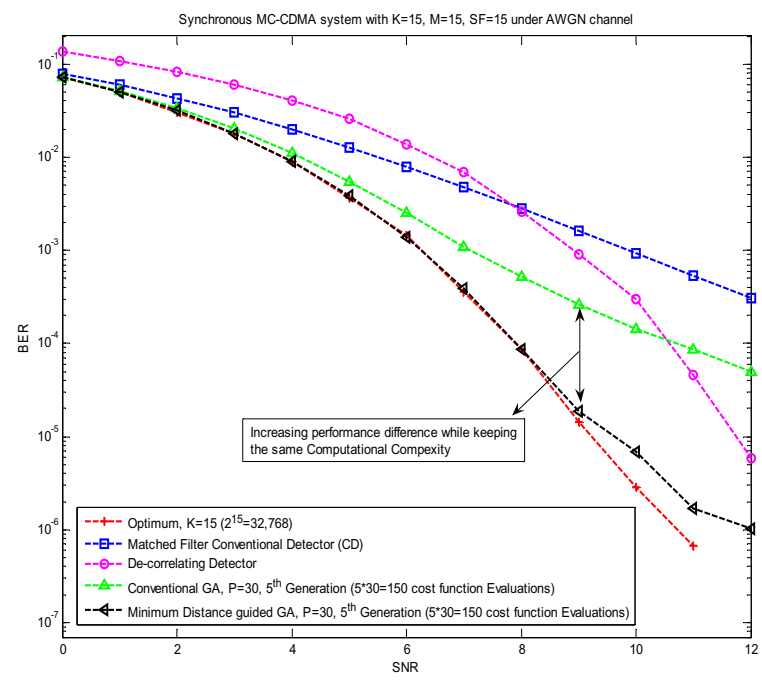

Fig. 9. BER Comparison of MDGA vs. Conventional GA [11] with the same computational complexity $(\mathrm{P}=30$ and $\mathrm{Gen}=5)$ vs. De-Correlating. The MDGA based MUD in 15-User synchronous MC-CDMA ( $\mathrm{SF}=15 \& \mathrm{M}=15)$ system, outperforms Conventional GA with significant reduction in the BER.

The relation between the population size and the number of users in MDGA helps us to further generalize the computational complexity in terms of number of users. For constant termination generation (e.g. Gen $=5$ ), the number of cost function evaluations are $P o p \times G e n=2 K \times 5=10 K$. Hence, the complexity becomes $\left(\frac{10 K}{2^{K}}\right) \times 100 \%$. The amount of computational complexity introduced is ten times the number of users, i.e. the number of evaluations $=10 K$, where $K$ $=\left(1,2,3, \ldots, \mathrm{K}_{\max }\right)$.

\section{CONCLUSIONS}

Our MDGA scheme carried out a balanced search based on an equal amount of exploration and exploitation within each step of the GA. The Minimum Distance technique is used effectively along with a novel replacement technique. The two techniques together have shown their significant improvement on the convergence rate as well as dramatic reduction on the computational complexity as compared to the existing GA assisted MUDs. Our scheme is less complex than the other genetic algorithms because it is realized based on simple genetic operators and features (standard bit mutation, one-point crossover \& fixed population size).

\section{REFERENCES}

[1] L. Hanzo, L.-L. Yang, E.-L. Kuan, and K. Yen, "Single- and Multi-Carrier CDMA: Multi-User Detection, Space-Time Spreading, Synchronization, Standards and Networking". Piscataway, NJ: IEEE Press/Wiley, 2003.

[2] R. Prasad; S. Hara, "Multicarrier Techniques For 4G Mobile Communications", IEEE Press/Wiley, June 2003.

[3] S. Hara and R. Prasad, "Overview of Multi-Carrier CDMA," IEEE Communications Magazine, vol. 35, pp. 126-133, Dec. 1997.

[4] S. Moshavi, "Multiuser Detection for DS-CDMA Communications", IEEE Communications Magazine, October 1996.

[5] S. Verdu, "Multiuser Detection", New York, USA: Cambridge University Press, 1998.

[6] S. L. Miller and B. J. Ranbolt, "MMSE Detection of Multi-carrier CDMA," IEEE Journal on Selected Areas in Communications, vol. 18, pp. 2356-2362, Nov 2000.

[7] P. Zong, K. Wang, and Y. Bar-Ness, "Partial Sampling MMSE Interference Suppression in Asynchronous Multicarrier CDMA System," IEEE Journal on Selected Areas in Communications, vol. 19, pp. 1605-1613, Aug 2001.

[8] M. Schnell and S. Kaiser, "Diversity Considerations for MC CDMA System in Mobile Communications," in Proceedings of IEEEISSSTA 1996, pp. 131-135,1996.

[9] J. H. Holland, "Adaptation in Natural and Artificial Systems", University of Michigan Press, Ann Arbor, 1975.

[10] M. J. Juntti, "Genetic Algorithms for Multiuser Detection in Synchronous CDMA," IEEE International Symposium on Inform. Theory, pp. 492, 1997.

[11] H. Wei, and L. Hanzo, "Reduced-Complexity Near-Optimum Genetic Assisted Multiuser Detection for Synchronous Multicarrier CDMA", Proc. of 2004 IEEE VTC-Spring Conf., Milan (I), May 17-19 2004, Vol. 3, pp. 1717-1721. 